\title{
Generalized compactness in linear spaces and its applications
}

\author{
V.Yu. Protasov*, M.E. Shirokov ${ }^{\dagger}$
}

\section{Contents}

1 Introduction $\quad 1$

2 On $\mu$-compact sets 3

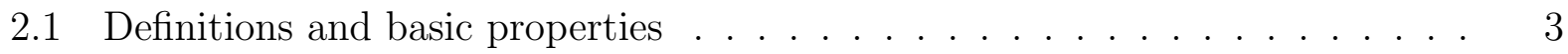

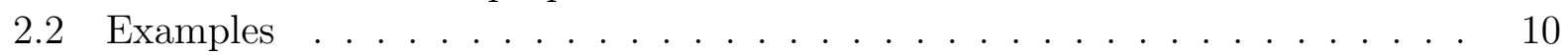

3 The CE-properties of $\mu$-compact convex sets

4 Applications to quantum information theory $\quad 18$

5 Possible generalizations and open questions $\quad 20$

6 Appendix $\quad 22$

6.1 The compactness criterion for subsets of the cone $\mathfrak{L}_{+}\left(\mathcal{H}, \mathcal{H}^{\prime}\right) \ldots \ldots . . . .22$

6.2 The openness criterion . . . . . . . . . . . . . . . 24

\section{Introduction}

Various properties and structure of compact sets in the convex analysis context have been studied thoroughly starting from the middle of the last century. An extensive bibliography is devoted to this theme (see [1, 2, 3] and references therein). The most important results are well known: the Krein-Milman theorem on convex hulls of extreme points, the Choquet theory on barycentric decompositions, properties of convex hulls (envelopes) of functions on convex compact sets. Some of the classical results have been extended to non-compact sets in locally convex spaces by Edgar [4, 5] and Bourgin [6, 7]. Such generalizations are interesting not only theoretically, but also very important in applications, for instance, in

*Lomonosov Moscow State University, e-mail:v-protassov@yandex.ru

†Steklov Mathematical Institute, e-mail:msh@mi.ras.ru 
mathematical physics [8], in quantum information theory [9], and so on. Of course, classical results of convex analysis cannot be extended to all non-compact sets. One has to postulate some special properties of these sets. The Choquet theory, for example, has been generalized to sets possessing the Radon-Nicodim property [4]. In [9] the several results on continuity of convex hulls of functions were extended to a special class of sets called $\mu$-compact sets. This class, characterized by the special relation between the topology and the structure of linear operations, is the main subject of this paper.

Problems of continuity of convex hulls of continuous functions (see definitions in the next section) have been studied in the literature since the 70 s of the last century. Under what conditions on the convex compact set $\mathcal{A}$ is the convex hull of any continuous (another assumption: concave continuous) function defined on $\mathcal{A}$ continuous? Vesterstrom in [10] showed that a necessary and sufficient condition for this is the openness of the barycenter map. He conjectured the equivalence of the continuity of the convex hull of any continuous concave function (this property was called by Lima the CE-property [11]) to the continuity of the convex hull of any continuous function (called in [9] the strong CE-property). This conjecture was proved by O'Brien in [12], who, moreover, showed the equivalence of the both CE-properties to the openness of the convex mixing map

$$
(x, y, \lambda) \mapsto \lambda x+(1-\lambda) y
$$

(the so-called "stability property for convex sets" [13, 14, 15]). The question arises if these results can be extended to non-compact sets $\mathcal{A}$. The first step towards the solution of this problem was made in [9], where so-called $\mu$-compact sets were defined. Some results on the CE-properties were generalized from compact sets to the class of $\mu$-compact sets. This, in particular, made it possible to derive several results concerning the entropic characteristics of infinite-dimensional quantum channels and systems.

In this paper we analyze the $\mu$-compactness property in detail, consider several examples that are important in applications, extend some classical results of convex analysis known earlier for compact sets only, in particular, the Vesterstrom-O'Brien theorem, to the class of $\mu$-compact sets.

The class of $\mu$-compact convex sets is defined by the requirement of weak compactness of preimages of all compact sets under the barycenter map. This property is not purely topological, it expresses certain relations between topology and the structure of linear operations. This class contains all compact sets as well as some important non-compact sets, for example, the set of density operators in a separable Hilbert space. $\mu$-compact sets do not possess many properties of compact sets, such as the boundedness of continuous functions, the Weierstrass theorem, and so on. Nevertheless, as we shall see, a lot of results of the Choquet theory and of the Vesterstrom-O'Brien theory can be extended to this class. Moreover, we give arguments showing that the class of $\mu$-compact sets is, in some sense, the largest class, to which the Vesterstrom-O'Brien theory can be extended.

This paper is organized as follows. In Section 2 we derive basic properties of $\mu$-compact sets. By a simple example we show that several results true for $\mu$-compact sets become false after a slight relaxing this assumption to pointwise $\mu$-compactness (this property is defined by the requirement of weak compactness of sets of measures with fixed barycenter). 
Further we consider examples of $\mu$-compact sets. We show, in particular, that the bounded part of the positive cone in the space $l_{p}$ for $p=1$ is $\mu$-compact, while for $p>1$ it is not even pointwise $\mu$-compact. The $\mu$-compactness of the set of Borel probability measures on a complete separable metric space is established. This result makes it possible to show that the convex closure operation respects the $\mu$-compactness property.

In Section 3 we complete the generalization of the Vesterstrom-O'Brien theorem to the class of $\mu$-compact sets, started in [9]. The $\mu$-compact version of the main result from [12] is proved. This establishes the equivalence of the continuity property for convex hulls of concave bounded continuous functions and the continuity property for convex hulls of arbitrary bounded continuous functions. We construct an example confirming our assumption that $\mu$-compact sets form the largest class of convex metrizable sets, for which this extension is possible. In Section 4 we apply some of our results to the quantum information theory. In Section 5 we discuss possible generalizations and formulate several open problems.

\section{On $\mu$-compact sets}

\subsection{Definitions and basic properties}

In Sections 2 and 3 we assume the set $\mathcal{A}$ to be a closed bounded subset of a locally convex space. We also suppose that the convex closure $\overline{\mathrm{co}} \mathcal{A}$ of $\mathcal{A}$ defined as the closure of its convex hull co $\mathcal{A}$ is a complete separable metric space. 1 We use the following notation:

$\operatorname{extr} \mathcal{A}$ is the set of extreme points of $\mathcal{A}$;

$C(\mathcal{A})$ is the set of continuous bounded functions on a set $\mathcal{A}$;

$P(\mathcal{A})$ and $Q(\mathcal{A})$ are the sets of convex and, respectively, concave continuous bounded functions on a convex set $\mathcal{A}$;

cof and $\overline{\mathrm{co}} f$ are the convex hull and the convex closure of a function $f$ on a convex set; they are defined as the maximal convex and the maximal convex closed (that is, lower semicontinuous) functions not exceeding $f$, respectively [3], [16];

$\mathfrak{P}_{n}=\left\{\left\{\pi_{i}\right\}_{i=1}^{n} \mid \pi_{i} \geq 0, \sum_{i=1}^{n} \pi_{i}=1\right\}$ is the simplex of all probability distributions with $n \leq+\infty$ outcomes.

Let $M(\mathcal{A})$ be a set of all Borel probability measures on the set $\mathcal{A}$ with the topology of weak convergence [17, 18].

With an arbitrary measure $\mu \in M(\mathcal{A})$ we associate its barycenter (average) $\mathbf{b}(\mu) \in \overline{\mathrm{co}} \mathcal{A}$, which is defined by the Pettis integral $([17,19])$

$$
\mathbf{b}(\mu)=\int_{\mathcal{A}} x \mu(d x) .
$$

\footnotetext{
${ }^{1}$ This means that the topology on the set $\overline{c o} \mathcal{A}$ is defined by a countable subset of the family of seminorms generating the topology of the entire locally convex space, and this set is separable and complete in the metric generated by this subset of seminorms.
} 
Let $M_{x}(\mathcal{A})$ be a convex closed subset of the set $M(\mathcal{A})$, which consists of measures $\mu$ such that $\mathbf{b}(\mu)=x \in \overline{\mathrm{co}} \mathcal{A}$.

We denote by $\left\{\pi_{i}, x_{i}\right\}$ the measure with a finite or countable number of atoms $\left\{x_{i}\right\}$ with weights $\left\{\pi_{i}\right\}$. Let $M^{\mathrm{f}}(\mathcal{A})$ and $M_{x}^{\mathrm{f}}(\mathcal{A})$ be subsets of the sets $M(\mathcal{A})$ and $M_{x}(\mathcal{A})$ respectively that consist of measures with finite supports.

The barycenter map

$$
M(\mathcal{A}) \ni \mu \mapsto \mathbf{b}(\mu) \in \overline{\mathrm{co}} \mathcal{A},
$$

is continuous, which can be shown easily by applying Prokhorov's theorem. Therefore, the image of any compact set in $M(\mathcal{A})$ under map (2) is compact in $\overline{\mathrm{co}} \mathcal{A}$. The inverse map $\mathbf{b}^{-1}$ may not possess this property. Generalizing the definition from $[9$ consider the class of convex sets, for which the map $\mathbf{b}^{-1}$ takes compact sets to compact sets.

Definition 1. The set $\mathcal{A}$ is called $\mu$-compact if the preimage of any compact subset of $\overline{\mathrm{co}} \mathcal{A}$ under barycenter map (2) is a compact subset of the set $M(\mathcal{A})$.

An arbitrary compact set is $\mu$-compact. Indeed, the compactness of $\mathcal{A}$ implies the compactness of $M(\mathcal{A})$ [18]. By using Prokhorov's theorem one can derive the following criterion of $\mu$-compactness [9].

Proposition 1. A convex set $\mathcal{A}$ is $\mu$-compact if and only if for any compact set $\mathcal{K} \subseteq \mathcal{A}$ and for any $\varepsilon>0$ there is a compact subset $\mathcal{K}_{\varepsilon} \subseteq \mathcal{A}$ such that for any $x \in \mathcal{K}$ and for any its expansion $x=\sum_{i=1}^{n} \lambda_{i} x_{i}$, where $\left\{x_{i}\right\}_{i=1}^{n} \subset \mathcal{A},\left\{\lambda_{i}\right\}_{i=1}^{n} \in \mathfrak{P}_{n}$, we have $\sum_{i: x_{i} \in \mathcal{A} \backslash \mathcal{K}_{\varepsilon}} \lambda_{i}<\varepsilon$

Proposition 1 and basic properties of the set $M(\mathcal{A})$ yield the following criterion of $\mu$-compactness, which is most convenient for applications.

Proposition 2. A convex set $\mathcal{A}$ is $\mu$-compact if and only if there is a family $F(\mathcal{A})$ of nonnegative concave functions on $\mathcal{A}$ with the following properties:

- the set $\{x \in \mathcal{A} \mid f(x) \leq c\}$ is relatively compact for any function $f \in F(\mathcal{A})$ and for any $c>0$;

- for any compact set $\mathcal{K} \subseteq \mathcal{A}$ there is a function $f \in F(\mathcal{A})$ such that $\sup _{x \in \mathcal{K}} f(x)<+\infty$.

Proof. The sufficiency easily follows from Proposition 1(see [9]). Let us prove the necessity. Let $\mathfrak{V}(\mathcal{A})$ be the set of lower semicontinuous functions $\varphi$ on $\mathcal{A}$ taking values in $[0,+\infty]$ and such that $\{x \in \mathcal{A} \mid \varphi(x) \leq c\}$ is compact for any $c \geq 0$. Applying Prokhorov's theorem (see [17], example 8.6.5, p. 236) we conclude that the set $M_{0} \subseteq M(\mathcal{A})$ is relatively compact if and only if there exists a function $\varphi \in \mathfrak{V}(\mathcal{A})$ such that

$$
\sup _{\mu \in M_{0}} \int_{\mathcal{A}} \varphi(x) \mu(d x)<+\infty .
$$

Consider the following family of concave nonnegative functions

$$
f_{\varphi}(x)=\sup _{\mu \in M_{x}(\mathcal{A})} \int_{\mathcal{A}} \varphi(y) \mu(d y), \varphi \in \mathfrak{V}(\mathcal{A}),
$$


on the set $\mathcal{A}$. This family possesses the first characteristic property of the family $F(\mathcal{A})$, which follows from the continuity of the barycenter map. The second property follows from the $\mu$-compactness of the set $\mathcal{A}$.

Remark 1. This is interesting that for all convex non-compact, but $\mu$-compact sets considered in Subsection 2.2 there exist families $F(\mathcal{A})$ that consist of affine lower semicontinuous functions.

There exists a criterion of $\mu$-compactness of a convex set in terms of properties of functions defined on this set [20]. More precisely, it is shown that the $\mu$-compactness is equivalent to the continuity of the operator of convex closure (that is, the double Fenchel transform) with respect to monotone pointwise converging sequences on the classes of continuous bounded and of lower semicontinuous lower bounded functions.

Let us note that continuous affine maps do not necessarily respect the $\mu$-compactness property. Nevertheless, we have the following simple consequence of Propositions 1 and 2 ,

Proposition 3. Let $\mathcal{A}$ and $\mathcal{B}$ be convex sets 2 and $\varphi$ be a continuous affine map from $\mathcal{A}$ to $\mathcal{B}$ such that for any compact set $\mathcal{C} \subseteq \mathcal{B}$ its preimage $\varphi^{-1}(\mathcal{C})$ is compact in $\mathcal{A}$. Then

$1)$ the $\mu$-compactness of $\mathcal{B}$ implies the $\mu$-compactness of $\mathcal{A}$;

2) if $\varphi$ is surjective, then the $\mu$-compactness of $\mathcal{A}$ implies the $\mu$-compactness of $\mathcal{B}$.

The operations of intersection, taking convex closure and Cartesian product respect $\mu$-compactness:

Proposition 4. 1) A closed subset of any $\mu$-compact set is $\mu$-compact.

2) The convex closure of any $\mu$-compact set is $\mu$-compact.

3) The Cartesian product of a finite or countable family of $\mu$-compact sets is $\mu$-compact (in the topology of coordinate-wise convergence).

Proof. 1) This follows directly from Definition 1 .

2) Combining the $\mu$-compactness of the set $\mathcal{A}$ and proposition 2 from [9] (its proof for our class of sets is literally the same as in that paper) we obtain that the barycenter map $\mu \mapsto \mathbf{b}(\mu)$ is a continuous affine surjection from $M(\mathcal{A})$ to $\overline{\mathrm{co}} \mathcal{A}$ satisfying the assumptions of Proposition 3. Applying the second part of that proposition and Corollary 4 from the next subsection we conclude that the set $\overline{\mathrm{co}} \mathcal{A}$ is $\mu$-compact.

3) By assertion 2) it suffices to consider the case of convex $\mu$-compact sets. Assume for every $n \in \mathbb{N}$ the set $\mathcal{A}^{n}$ is $\mu$-compact. Let us show that the set $\mathcal{A}=\otimes_{n \in \mathbb{N}} \mathcal{A}^{n}$ is $\mu$-compact in the topology of coordinatewise convergence. For an arbitrary compact set $\mathcal{K} \subset \mathcal{A}$ and for each $n \in \mathbb{N}$ let $\mathcal{K}^{n}$ be the projection of $\mathcal{K}$ onto $\mathcal{A}^{n}$. The set $\mathcal{K}^{n}$ consists of points $x^{n} \in \mathcal{A}^{n}$ which are the corresponding coordinates of some point $x \in \mathcal{K}$. This set is compact. Since the set $\mathcal{A}^{n}$ is $\mu$-compact, it follows from Proposition 1 that for any $\varepsilon>0$ there exists the corresponding compact $\mathcal{K}_{\varepsilon}^{n} \subset \mathcal{A}^{n}$. Since $\mathcal{K} \subseteq \otimes_{n \in \mathbb{N}} \mathcal{K}^{n}$, we have $\mathcal{K}_{\varepsilon}=\otimes_{n \in \mathbb{N}} \mathcal{K}_{\varepsilon 2^{-n}}^{n}$. It is easy to check that this set satisfies the assumptions of Proposition 11. Therefore, the set $\mathcal{A}$ is $\mu$-compact.

\footnotetext{
${ }^{2}$ It is assumed that the set $\mathcal{B}$ possess all the properties mentioned in the beginning of Subsection 2.1 .
} 
The first assertion of Proposition 4 implies that the intersection of $\mu$-compact sets is $\mu$-compact. However, their union or Minkowski sum is, in general, not $\mu$-compact (Remark (4).

Remark 2. By the second assertion of Proposition 4 to prove the $\mu$-compactness of a convex set $\mathcal{A}$ it suffices to show the $\mu$-compactness of any its subset $\mathcal{B}$ such that $\mathcal{A}=\overline{\mathrm{co}} \mathcal{B}$. Note that the sets of measures $M(\mathcal{A})$ and $M(\mathcal{B})$ (that are involved in the definition of $\mu$-compactness of the sets $\mathcal{A}$ and $\mathcal{B}$ ) can be completely different. For example, if $\mathcal{A}$ is a simplex, then $\mathcal{B}$ can be a countable family $\left\{e_{i}\right\}$ of isolated extreme points of the set $\mathcal{A}$. Hence $M(\mathcal{B})$ is isomorphic to the set $\mathfrak{P}_{+\infty}$ of all probability distributions with countable number of outcomes. The criterion of $\mu$-compactness for the set $\mathcal{B}$, and therefore, for the set $\mathcal{A}$, can be formulated as follows: for any compact set $\mathcal{K} \subset \mathcal{A}$ and for any $\varepsilon>0$ there exists $n$ such that the inclusion $\sum_{i=1}^{+\infty} \pi_{i} e_{i} \in \mathcal{K},\left\{\pi_{i}\right\} \in \mathfrak{P}_{+\infty}$, implies $\sum_{i=n+1}^{+\infty} \pi_{i}<\varepsilon$.

The second assertion of Proposition 4 together with Proposition 5 below lead to the following observation. Let $\mathcal{A}$ be a $\mu$-compact convex set in the initial topology $\tau$ and let $\tau^{\prime}$ be a stronger topology on $\mathcal{A}$, that coincides with $\tau$ on the set $\overline{\operatorname{extr} \mathcal{A}}$; then the set $\mathcal{A}$ is $\mu$-compact in the topology $\tau^{\prime}$.

Proposition 3 implies that all isomorphisms in the category of $\mu$-compact convex sets are affine homeomorphisms. The affinity assumption cannot be omitted, which is seen from the following example. Suppose $\mathcal{A}$ is a convex set that is not $\mu$-compact. The first assertion of Proposition 4 and Corollary 4 from the next subsection yield that the subset of the set $M(\mathcal{A})$ that consists of Dirac (single-atomic) measures is a $\mu$-compact set, which is, moreover, homeomorphic to the set $\mathcal{A}$. This observation shows that the $\mu$-compactness property, in contrast to the compactness, is not purely topological. It is defined by composition of the topology and of the structure of the operation of convex mixing.

Proposition 3 gives the following condition of $\mu$-compactness of families of maps. This condition is applied in the next section.

Corollary 1. Let $\mathfrak{F}(\mathcal{X}, \mathcal{Y})$ be a locally convex space with the topology $\tau$ of maps from the set $\mathcal{X}$ to a locally convex space $\mathcal{Y}$. Let also $\mathfrak{F}_{0}$ be a convex closed bounded subset of the space $\mathfrak{F}(\mathcal{X}, \mathcal{Y})$ that consists of maps taking values from the convex $\mu$-compact set $\mathcal{A} \subset \mathcal{Y}$. Moreover, $\mathfrak{F}_{0}$ is a complete separable metric space, for which there is an element $x_{0} \in \mathcal{X}$ such that:

1) $\left\{\tau-\lim _{n \rightarrow+\infty} \Phi_{n}=\Phi_{0}\right\} \Rightarrow\left\{\lim _{n \rightarrow+\infty} \Phi_{n}\left(x_{0}\right)=\Phi_{0}\left(x_{0}\right)\right\}, \quad \forall\left\{\Phi_{n}\right\} \subset \mathfrak{F}_{0}$;

2) the set $\left\{\Phi \in \mathfrak{F}_{0} \mid \Phi\left(x_{0}\right) \in \mathcal{C}\right\}$ is relatively $\tau$-compact for any compact set $\mathcal{C} \subseteq \mathcal{A}$.

Then the set $\mathfrak{F}_{0}$ is $\mu$-compact.

Proof. The continuous affine map

$$
\mathfrak{F}_{0} \ni \Phi \mapsto \Phi\left(x_{0}\right) \in \mathcal{A}
$$

satisfies the assumptions of Proposition 3. The first part of that proposition implies the $\mu$-compactness of the set $\mathfrak{F}_{0}$. 
For further analysis of the $\mu$-compactness we need a weaker version of this property.

Definition 2. The set $\mathcal{A}$ is called pointwise $\mu$-compact if for any $x \in \overline{\mathrm{co}} \mathcal{A}$ the set $M_{x}(\mathcal{A})$ is a compact subset of the set $M(\mathcal{A})$.

Clearly, the pointwise $\mu$-compactness follows from the $\mu$-compactness. However, as we shall see in Proposition 13, these two properties are not equivalent.

$\mu$-compact sets do not possess many properties of compact sets, such as uniform continuity and boundedness of continuous functions, the Weierstrass theorem of extremal values of continuous functions, and so on. It appears, however, that $\mu$-compact sets inherit some important properties of compact sets. This allows us to extend many results of the Choquet theory and of the Vesterstrom-O'Brien theory to those sets (see the next section).

If $\mathcal{A}$ is a convex set, then one can introduce the following partial order, called the Choquet order, on the set $M(\mathcal{A})[1],[6]$. We suppose that $\mu \succ \nu$ if and only if

$$
\int_{\mathcal{A}} f(y) \mu(d y) \geq \int_{\mathcal{A}} f(y) \nu(d y)
$$

for any function $f \in P(\mathcal{A})$.

A measure $\mu \in M(\mathcal{A})$ is called maximal if $\nu \succ \mu$ implies $\nu=\mu$ for any measure $\nu \in M(\mathcal{A})$. If $\mu$ and $\nu$ are measures from $M(\mathcal{A})$ such that $\mu \succ \nu$, then $\mathbf{b}(\mu)=\mathbf{b}(\nu)$. This follows from the fact that the set of continuous bounded affine functions on the set $\mathcal{A}$ separates its points. The following fact is a straightforward consequence of Definition 2.

Lemma 1. Let $\mathcal{A}$ be a convex pointwise $\mu$-compact set. Any subset of the set $M(\mathcal{A})$ that is linearly ordered by the relation $\prec$ has the least upper bound.

Proof . Any subset of the set $M(\mathcal{A})$ linearly ordered by the relation $\prec$, which is actually a net $\left\{\mu_{\lambda}\right\}_{\lambda \in \Lambda}$, is contained in $M_{x}(\mathcal{A})$ for some $x \in \mathcal{A}$ and, consequently, is relatively compact. Whence there is a subnet $\left\{\mu_{\lambda_{\pi}}\right\}_{\pi \in \Pi}$ that converges to some measure $\mu_{0} \in M_{x}(\mathcal{A})$. One can easily verify that $\mu_{\lambda} \prec \mu_{0}$ for all $\lambda \in \Lambda$.

Combining Lemma 1 with theorem 2.4 from [5] we see that the class of convex pointwise $\mu$-compact sets is a proper subclass of the class of convex sets with the Radon-Nicodim property. This class is studied in an extensive literature [4, 5, 6].

Applying Lemma 1 and Zorn's lemma one can easily prove the following generalizations of the Krein-Milman theorem and of the Choquet theorem to pointwise $\mu$-compact sets. This actually follows from the Radon-Nicodim property of these sets [5].

Proposition 5. Let $\mathcal{A}$ be a pointwise $\mu$-compact convex set. Then $\overline{\operatorname{co}}(\operatorname{extr} \mathcal{A})=\mathcal{A}$ and $\mathbf{b}(M(\overline{\operatorname{extr} \mathcal{A}}))=\mathcal{A}$.

Proof. Since the set of finitely supported measures is dense in $M(\overline{\operatorname{extr} \mathcal{A}})$ [18, theorem 6.3$]$, we see that the first assertion follows from the second one.

Let $x_{0} \in \mathcal{A}$. Lemma 1 combined with Zorn's lemma yields the existence of the maximal measure $\mu_{*}$ in $M_{x_{0}}$. From the properties of the Choquet ordering it follows that the measure 
$\mu_{*}$ is also maximal in $M(\mathcal{A})$; hence it lies in $M(\overline{\operatorname{extr} \mathcal{A}})$ (see the proof of theorem 5.2 in [4]). Thus, $x_{0} \in \mathbf{b}(M(\overline{\operatorname{extr} \mathcal{A}}))$.

Another property inherited by $\mu$-compact sets from compact ones is the following representation of convex closure of lower semicontinuous functions [9, proposition 3]. Its proof is easily generalized to our class of sets.

Proposition 6. If $f$ is a lower semicontinuous lower bounded function on a convex $\mu$-compact set $\mathcal{A}$, then its convex closure can be determined by the expression

$$
\overline{\mathrm{co}} f(x)=\inf _{\mu \in M_{x}(\mathcal{A})} \int_{\mathcal{A}} f(y) \mu(d y), \quad \forall x \in \mathcal{A},
$$

where the infimum is attained at some measure $\mu_{x}^{f}$ from $M_{x}(\mathcal{A})$.

This representation is a crucial point for most results on convex closures of functions defined on $\mu$-compact sets.

If $f$ is a continuous bounded function on a convex $\mu$-compact set $\mathcal{A}$, then combining the continuity of the functional $M(\mathcal{A}) \ni \mu \mapsto \int_{\mathcal{A}} f(x) \mu(d x)$ and [9, lemma 1] we see that the infimum in (3) can be taken over finitely-supported measures.

Corollary 2. An arbitrary continuous bounded function $f$ on a convex $\mu$-compact set $\mathcal{A}$ possesses a lower semicontinuous (closed) convex hull, that is,

$$
\operatorname{cof}(x)=\inf _{\left\{\pi_{i}, x_{i}\right\} \in M_{x}^{\mathrm{f}}(\mathcal{A})} \sum_{i} \pi_{i} f\left(x_{i}\right)=\overline{\operatorname{co}} f(x), \quad \forall x \in \mathcal{A} .
$$

This is a $\mu$-compact generalization of corollary I.3.6 in [2]. The $\mu$-compactness assumption about the set $\mathcal{A}$ in Proposition 6 and in Corollary 2 cannot be weakened to pointwise $\mu$-compactness.

Proposition 7. If a convex set $\mathcal{A}$ is not $\mu$-compact, but pointwise $\mu$-compact, then there exists a continuous bounded function $f$ on $\mathcal{A}$, whose convex hull cof is not lower semicontinuous. This means that representation (3) for the convex closure $\overline{\mathrm{co}} f$ of the function $f$ does not hold.

Proof. Since the set $\mathcal{A}$ is not $\mu$-compact, there exists a non-compact sequence of measures $\left\{\mu_{n}\right\} \subset M(\mathcal{A})$ such that the corresponding sequence $\left\{x_{n}=\mathbf{b}\left(\mu_{n}\right)\right\} \subset \mathcal{A}$ converges. By Prokhorov's theorem the sequence $\left\{\mu_{n}\right\}$ is not tight. As it was shown in the proof of theorem 8.6.2 in [17] this guarantees the existence of $\varepsilon>0$ and $\delta>0$ such that for any compact set $\mathcal{K} \subset \mathcal{A}$ and any natural $N$ there is $n>N$, for which $\mu_{n}\left(U_{\delta}(\mathcal{K})\right)<1-\varepsilon$, where $U_{\delta}(\mathcal{K})$ is a closed $\delta$-neighborhood of the compact set $\mathcal{K}$. Since finitely supported measures is dense in the set of all measures with a fixed barycenter [9, lemma 1], applying corollary 8.2.9 from [17], we may assume that the sequence $\left\{\mu_{n}\right\}$ contains only finitely supported measures.

Let $x_{0}$ be a limit of the sequence $\left\{x_{n}\right\}$. By the pointwise $\mu$-compactness of the set $\mathcal{A}$ for the value $\varepsilon$ defined above there exists a convex compact set $\mathcal{K}_{\varepsilon}$ such that $\mu\left(\mathcal{K}_{\varepsilon}\right) \geq 1-\varepsilon / 2$ for any measure $\mu \in M_{x_{0}}(\mathcal{A})$. 
Let $f$ be a bounded continuous function on $\mathcal{A}$ such that $f(x)=1$ for all $x \in \mathcal{K}_{\varepsilon}$ and $f(x) \leq 0$ for every $x \in \mathcal{A} \backslash U_{\delta}\left(\mathcal{K}_{\varepsilon}\right)$. Then from the properties of the sequences $\left\{x_{n}\right\}$ and $\left\{\mu_{n}\right\}$ it follows that for any natural $N$ there is $n>N$, for which $\operatorname{cof}\left(x_{n}\right)<1-\varepsilon$. On the other hand, the properties of the set $M_{x_{0}}(\mathcal{A})$ imply that $\operatorname{cof}\left(x_{0}\right) \geq 1-\varepsilon / 2$. Thus, the function cof is not lower semicontinuous.

Remark 3. The pointwise $\mu$-compactness condition cannot be omitted in the proof of Proposition 17. This shows that $\mu$-compact sets do not form the maximal class of convex sets, for which Corollary 2 holds. However, without the pointwise $\mu$-compactness Corollary 2 may fail (see Example 1 in the next section).

To construct an example showing that the pointwise $\mu$-compactness condition is essential in Proposition 17, we consider the unit ball $\mathcal{B}$ in a separable Hilbert space. Clearly, that is not a pointwise $\mu$-compact set. Let us show that for any continuous bounded function $f$ on $\mathcal{B}$ its convex hull cof is continuous. Since a bounded convex function is continuous at interior points of its domain, it suffices to prove the continuity of co $f$ on the boundary of $\mathcal{B}$, that is, on the unit sphere.

Let $x$ be a point of the unit sphere. For arbitrary $\varepsilon>0$ there exists $\delta>0$ such that $|f(x)-f(y)|<\varepsilon$, whenever $\|x-y\|<2 \delta$. Applying Lemma 2 stated below, we conclude that for any $z$ such that $\|z-x\|<\delta$ and for any $\mu \in M_{z}(\mathcal{B})$ the measure of a ball of radius $2 \delta$ centered at $x$ is at least $r(\delta, z)$. Therefore, the value $\left|f(x)-\int_{\mathcal{B}} f(t) d \mu\right|$ does no exceed $\varepsilon r(\delta, z)+N(1-r(\delta, z))$, where $N=\sup _{t \in \mathcal{B}}|f(t)|$. Whence

$$
|f(x)-\operatorname{cof}(z)| \leq \varepsilon r(\delta, z)+N(1-r(\delta, z)) .
$$

Since for $z \rightarrow x$ we have $\|z\| \rightarrow 1$, it follows that $r(\delta, z) \rightarrow 1$ and so $\operatorname{cof}(z) \rightarrow f(x)$. It remains to note that $\operatorname{cof}(x)=f(x)$, because $x$ is an extreme point of $\mathcal{B}$.

Lemma 2. Let $\mathcal{B}$ be the unit ball of the Hilbert space and let $\delta>0$. For an arbitrary point $z \in \mathcal{B}$ such that $\|z\|>1-\delta$ and for any measure $\mu \in M_{z}(\mathcal{B})$ the measure of a ball of radius $\delta$ centered at $z$ is at least $r(\delta, z)=\frac{\delta^{2}-\left(1-\|z\|^{2}\right)}{\delta^{2}-(1-\|z\|)^{2}}$.

Proof. Let $\bar{z}=\frac{z}{\|z\|}$ and

$$
\mathcal{B}_{0}=\left\{y \in \mathcal{B},(y, \bar{z})<\frac{1+\|z\|^{2}-\delta^{2}}{2\|z\|}\right\}, \quad \mathcal{B}_{1}=\left\{y \in \mathcal{B},(y, \bar{z}) \geq \frac{1+\|z\|^{2}-\delta^{2}}{2\|z\|}\right\} .
$$

By a direct calculation we show that if $\|y\|=1$ and $\|y-z\|>\delta$, then $y \in \mathcal{B}_{0}$. Consequently, all points of the ball $\mathcal{B}$ lying at a distance more than $\delta$ from the point $z$ are located in the set $\mathcal{B}_{0}$. Denote by $c_{i}$ the barycenter of the measure $\mu$ on the set $\mathcal{B}_{i}, i=0,1$. We have $z=\mu\left(\mathcal{B}_{0}\right) c_{0}+\mu\left(\mathcal{B}_{1}\right) c_{1}$. It is obvious that $\left(c_{0}, \bar{z}\right) \leq \frac{1+\|z\|^{2}-\delta^{2}}{2\|z\|}$ and $\left(c_{1}, \bar{z}\right) \leq 1$. Therefore,

$$
\|z\|=(z, \bar{z})<\left(1-\mu\left(\mathcal{B}_{1}\right)\right) \frac{1+\|z\|^{2}-\delta^{2}}{2\|z\|}+\mu\left(\mathcal{B}_{1}\right),
$$

which gives the desired inequality for the measure of $\mathcal{B}_{1}$, and hence for the measure of the ball of radius $\delta$ centered at $z$, since this ball contains $\mathcal{B}_{1}$. 
One of the most important conclusions from Proposition 6 is that any lower semicontinuous lower bounded function $f$ defined on a convex $\mu$-compact set $\mathcal{A}$ coincides with its convex closure $\overline{\mathrm{co}} f$ on the set of extreme points $\operatorname{extr} \mathcal{A}$. Furthermore, that proposition enables us to obtain the following representation for the $\operatorname{set} \operatorname{extr} \mathcal{A}$, which will help us in Section 3 .

Proposition 8. Let $\mathcal{A}$ be a $\mu$-compact convex set. Then

$$
\operatorname{extr} \mathcal{A}=\bigcap_{f \in Q(\mathcal{A})} \mathcal{B}_{f}, \quad \text { where } \quad \mathcal{B}_{f}=\{x \in \mathcal{A} \mid f(x)=\overline{\operatorname{co}} f(x)\}
$$

Proof. The inclusion extr $\mathcal{A} \subseteq \mathcal{B}_{f}$ for any function $f \in Q(\mathcal{A})$ follows from (4). Suppose $x_{0} \in \mathcal{A} \backslash \operatorname{extr} \mathcal{A}$; then there are two distinct points $x_{1}$ and $x_{2}$ in $\mathcal{A}$ such that $x_{0}=\frac{1}{2} x_{1}+\frac{1}{2} x_{2}$. To prove that $x_{0}$ is not in $\bigcap_{f \in Q(\mathcal{A})} \mathcal{B}_{f}$ one needs to find a function $f$ from $Q(\mathcal{A})$ such that $f\left(x_{0}\right)>\frac{1}{2} f\left(x_{1}\right)+\frac{1}{2} f\left(x_{2}\right)$. The function $-a^{2}(\cdot)$ will do, where $a$ is an affine continuous bounded function on $\mathcal{A}$ such that $a\left(x_{1}\right) \neq a\left(x_{2}\right)$.

Example 1 in the next subsection shows the importance of the $\mu$-compactness assumption in Proposition 8 .

Arguing as in the proof of Proposition 7 one can easily produce the following necessary condition for representation (5). This is a condition of local $\mu$-compactness in neighborhood of the set $\operatorname{extr} \mathcal{A}$ : for any extreme point $x_{0}$ of the set $\mathcal{A}$ and for any sequence $\left\{x_{n}\right\} \subset \mathcal{A}$ converging to $x_{0}$ the set $\mathbf{b}^{-1}\left(\left\{x_{n}\right\}\right)$ is compact in $M(\mathcal{A})$.

\section{$2.2 \quad$ Examples}

Any compact set is obviously $\mu$-compact. In this section we consider the several most important examples of $\mu$-compact, but not compact sets.

Proposition 9. The bounded part3 of the positive cone in the space $l_{1}$ is $\mu$-compact.

Proof. It is sufficient to take the family of functions of the form $l_{1} \ni\left\{x_{i}\right\} \mapsto \sum_{i} h_{i} x_{i}$, where $\left\{h_{i}\right\}$ is an increasing unbounded sequence of positive numbers, and then apply Proposition 2 taking into account the compactness criterion for subsets of the space $l_{1}$.

Corollary 3. The set $\mathfrak{P}_{+\infty}$ of all probability distributions with countably many outcomes is a $\mu$-compact subset of the space $l_{1}$.

Remark 4. Let $\mathcal{A}_{1}=\left\{x \in l_{1} \mid x \geq 0,\|x\|_{1} \leq 1\right\}$. Proposition 9 implies that the sets $\mathcal{A}_{1}$ and $-\mathcal{A}_{1}$ are both $\mu$-compact in the metric $l_{1}$. However, neither their convex hull nor their Minkowski sum is $\mu$-compact. They are actually not even pointwise $\mu$-compact, because they contain a unit ball of the space $l_{1}$, which is not pointwise $\mu$-compact (this is easy to show).

\footnotetext{
${ }^{3}$ Here and in what follows by the bounded part of a positive cone in an ordered Banach space we mean the intersection of this cone with a unit ball.
} 
Proposition 10. An arbitrary weakly closed bounded (in variation) set of Borel measures on a complete separable metric space is $\mu$-compact in the weak convergence topology.

Proof. Let $X$ be a complete separable metric space, $M$ be a weakly closed bounded set of Borel measures on that space $X, \mathfrak{V}(X)$ be the set of all lower semicontinuous functions $\varphi$ on $X$ taking values from $[0,+\infty]$ such that the set $\{x \in X \mid \varphi(x) \leq c\}$ is compact for all $c \geq 0$. Prokhorov's theorem (see [17], example 8.6.5, p. 236) implies that the set $M_{0} \subseteq M$ is relatively compact if and only if there exists a function $\varphi \in \mathfrak{V}(X)$, for which

$$
\sup _{\mu \in M_{0}} \int_{X} \varphi(x) \mu(d x)<+\infty \text {. }
$$

Hence the family of affine lower semicontinuous functions $f_{\varphi}(\mu)=\int_{X} \varphi(x) \mu(d x), \varphi \in \mathfrak{V}(X)$ on the set $M$ satisfies all the assumptions of Proposition 2 ,

Corollary 4. The set of all Borel probability measures on a complete separable metric space is $\mu$-compact in the topology of weak convergence.

Using Propositions 3 and 9 as well as Proposition [15 stated below one can prove the following assertion on the properties of the cone of positive operators in the Shatten class of order $p$, that is, in the Banach space of all operators acting in a separable Hilbert space $\mathcal{H}$ such that $\operatorname{Tr}|A|^{p}<+\infty$ with the norm $\|A\|_{p}=\left(\operatorname{Tr}|A|^{p}\right)^{1 / p}$.

Proposition 11. The bounded part of the positive cone in the Shatten class of order $p$ is $\mu$-compact precisely for $p=1$.

Proof. In the case $p=1$ by applying the compactness criterion for subsets of the positive cone $\mathfrak{T}_{+}(\mathcal{H})$ of the space of trace class operators $\mathfrak{T}(\mathcal{H})([21]$, Appendix), we obtain that the map taking an operator $A \in \mathfrak{T}_{+}(\mathcal{H})$ to the sequence of its diagonal elements (in some fixed basis of the space $\mathcal{H}$ ) satisfies the assumptions of the first part of Proposition 3 (with the bounded parts of the positive cones of the spaces $\mathfrak{T}(\mathcal{H})$ and $l_{1}$ in the roles of $\mathcal{A}$ and $\mathcal{B}$ respectively). Combining that proposition with Proposition 9 we obtain the $\mu$-compactness of the bounded part of the cone $\mathfrak{T}_{+}(\mathcal{H})$.

The cone of positive operators in the Shatten class of order $p>1$ contains a subcone of commuting operators. That subcone is affinely homeomorphic to $\mathcal{A}_{p}$ (the bounded part of the positive cone in the space $l_{p}$ ), which is not $\mu$-compact (Proposition [15).

Proposition 11 yields, in particular, the $\mu$-compactness of the set $\mathfrak{S}(\mathcal{H})$ of quantum states - density operators in a separable Hilbert space $\mathcal{H}$. This fact was originally proved in [22]. Corollary 1 and Lemma 5 from Appendix imply the following result.

Proposition 12. Let $\mathfrak{L}_{+}\left(\mathcal{H}, \mathcal{H}^{\prime}\right)$ be the cone of positive linear continuous maps from the Banach space $\mathfrak{T}(\mathcal{H})$ of trace class operators in a separable Hilbert space $\mathcal{H}$ into a similar space $\mathfrak{T}\left(\mathcal{H}^{\prime}\right)$. Then the bounded (in the operator norm) part of this cone is $\mu$-compact in the strong operator topology.

Proposition 12 implies $\mu$-compactness of the sets of quantum operations and of quantum channels in the topology of strong convergence [21]. 
Proposition 12 also yields the $\mu$-compactness (in the strong operator topology) of the bounded part of the cone of linear continuous positive operators in $l_{1}$. Indeed, this cone is naturally identified with a subset of the cone $\mathfrak{L}_{+}\left(\mathcal{H}, \mathcal{H}^{\prime}\right)$. This shows the $\mu$-compactness of the set of sub-Markov operators, in particular, Markov operators.

Now consider some "negative" examples. Proposition 13 gives an example of pointwise $\mu$-compact sets (Definition 2) that are not $\mu$-compact. We are going to see that in Hilbert space there are no $\mu$-compact sets that are not compact (Proposition 14). Then we give several examples of sets that are not even pointwise $\mu$-compact.

By definition the pointwise $\mu$-compactness property of a set survives weakening the topology. The next proposition shows that $\mu$-compactness does not possess this property. The bounded part of the positive cone in $l_{1}$ loses the $\mu$-compactness after weakening the topology.

Proposition 13. For any $p>1$ the simplex $\Delta_{p}=\left\{x \in l_{p} \mid x \geq 0, \sum_{i=1}^{+\infty} x^{i} \leq 1\right\}$ in the space $l_{p}$ is pointwise $\mu$-compact, but not $\mu$-compact.

Proof. The pointwise $\mu$-compactness of the set $\Delta_{p}$ follows from the remark above. Let us show that $\Delta_{p}$ is not $\mu$-compact in the space $l_{p}$. Consider an increasing sequence of natural numbers $\left\{n_{r}\right\}_{r \in \mathbb{N}}$ and a sequence of nonnegative numbers $\left\{z_{i}\right\}_{i \in \mathbb{N}}$ such that $\sum_{i=n_{r}}^{n_{r+1}-1} z_{i}=1$ and $\sum_{i=n_{r}}^{n_{r+1}-1}\left(z_{i}\right)^{p} \leq \frac{1}{r}$ for each $r \geq 1$. The set $\mathcal{K}=\left\{y \in \Delta_{p} \mid \forall r \in \mathbb{N} \sum_{i=n_{r}}^{+\infty}\left(y^{i}\right)^{p} \leq \frac{1}{r}\right\}$ is compact. If the set $\Delta_{p}$ is $\mu$-compact, then by Proposition 1 for any $\varepsilon>0$ there is the corresponding compact set $\mathcal{K}_{\varepsilon}$. The set $\mathcal{K}_{\varepsilon}$ can contain only a finite number of vectors of the canonical basis $\left\{e_{i}\right\}$, otherwise it is not compact. Let $N$ be such that $e_{i} \notin \mathcal{K}_{\varepsilon}$ for $i>N$. Take an arbitrary $r$ for which $n_{r}>N$ and denote by $x \in \Delta_{p}$ the vector with coordinates $x^{i}=z^{i}$ for $n_{r} \leq i<n_{r+1}$ and $x^{i}=0$ for all other $i$. Clearly, $x \in \mathcal{K}$. Since $x=\sum_{i=n_{r}}^{n_{r+1}-1} x^{i} e_{i}$ and $\sum_{i=n_{r}}^{n_{r+1}-1} x^{i}=1$, but $e_{i} \notin \mathcal{K}_{\varepsilon}$ for all $i=\overline{n_{r}, n_{r+1}-1}$, we come to the contradiction with the $\mu$-compactness criterion from Proposition 1.

In particular, Proposition 13 shows that a Hilbert space contains noncompact pointwise $\mu$-compact sets. It appears that it does not contain $\mu$-compact sets that are not compact.

Proposition 14. There are no $\mu$-compact subsets of Hilbert space that are not compact. Proof. We describe the idea of the proof omitting details, which can be easily reconstructed by the reader. Let $\mathcal{A}$ be a bounded convex closed subset of a Hilbert space. Without loss of generality it can be assumed that its diameter is 1 . If $\mathcal{A}$ is not compact, then there exists a sequence of its elements $\left\{a_{k}\right\}_{k \in \mathbb{N}}$ such that all their norms and all pairwise distances between them exceed some value $\varepsilon>0$. Because of the weak compactness of $\mathcal{A}$ it can be assumed, after possible passing to a subsequence, that the sequence $\left\{a_{k}\right\}$ converges weakly to some element $a$. Since the set $\mathcal{A}$ is convex and closed, we have $a \in \mathcal{A}$. The sequence $\left\{b_{k}=a_{k}-a\right\}_{k \in \mathbb{N}}$ converges weakly to zero. Hence by passing to subsequences one can achieve a fast convergence to zero for the scalar products: $\left(b_{i}, b_{j}\right) \leq \varepsilon^{2} 2^{-i-j}$ for every $i \neq j$. Since $\varepsilon \leq\left\|b_{i}\right\|_{2} \leq 1$ for all $i \in \mathbb{N}$, invoking the Cauchy-Schwarz inequality we 
conclude that any sequence $x \in l_{2}$ satisfies the inequalities

$$
\frac{\varepsilon}{2}\|x\|_{2} \leq\left\|\sum_{i} x^{i} b_{i}\right\|_{2} \leq 2\|x\|_{2} .
$$

This means that the system of elements $\left\{b_{k}\right\}$ possesses the Riesz basis property. Then there is a continuous linear operator that is continuously invertible and taking the system $\left\{b_{k}\right\}$ to an orthonormal one [23. This operator maps the convex hull of points $a$ and $\left\{a_{k}\right\}_{k \in \mathbb{N}}$ to the set $\Delta_{2}$ from Proposition [13, which is not $\mu$-compact. Hence, this convex hull is not $\mu$-compact, therefore nor is the set $\mathcal{A}$.

Let us now consider two important examples of not pointwise $\mu$-compact sets.

Proposition 15. For every $p>1$ the set $\mathcal{A}_{p}=\left\{x \in l_{p} \mid x \geq 0,\|x\|_{p} \leq 1\right\}$ (the bounded part of the positive cone in the space $\left.l_{p}\right)$ is not pointwise $\mu$-compact.

Proof. Let us show that if a point $x \in \mathcal{A}_{p}$ is such that $\|x\|_{p}<\frac{1}{3}$ and $\sum_{i} x_{i}=+\infty$, then it has no compact set $\mathcal{K}_{\varepsilon}$ for $\varepsilon=\frac{1}{3}$ (see Proposition 1). If such a compact set exists, then it can contain only finitely many elements of the canonical basis $\left\{e_{i}\right\}$. Take a sufficiently large $N$ such that $e_{i} \notin \mathcal{K}_{\varepsilon}$ for every $i>N$. Since the series $\sum_{i} x_{i}$ diverges, we see that there exists $r$, for which $s=\sum_{i=N+1}^{N+r} x^{i} \in\left(\frac{1}{3}, \frac{2}{3}\right)$. Let $\bar{x}=x-\sum_{i=N+1}^{N+r} x^{i} e_{i}$ (the entries of the vector $\bar{x}$ from the $(N+1)$ st to the $(N+r)$ th are zeros, and the others coincide with the corresponding entries of $x)$. Then $x=(1-s)\left(\frac{1}{1-s} \bar{x}\right)+\sum_{i=N+1}^{N+r} x^{i} e_{i}$. Since $\frac{1}{1-s}<3$ and $\|\bar{x}\|_{p}<\frac{1}{3}$, it follows that $\frac{1}{1-s} \bar{x} \in \mathcal{A}_{p}$. All the points $e_{i}$ in this barycentric combination lie outside of $\mathcal{K}_{\varepsilon}$, but their total weight $s$ exceeds $\frac{1}{3}$, which is a contradiction.

The next example complements Proposition 7 and demonstrates that not pointwise $\mu$-compact sets do not have to satisfy relations (3) and (41) for continuous bounded concave functions. This violates representation (5)).

Example 1. Let $f$ be a continuous function on the bounded part $\mathcal{A}_{p}$ of the positive cone of the space $l_{p}$ for $p>1$ that takes the value 1 at zero and vanishes at all the vectors of the canonical basis $\left\{e_{n}\right\}$ of the space $l_{p}$. For example, the function $f(\cdot)=1-\|\cdot\|_{p}$. Since the zero vector of the space $l_{p}$ is a limit point of the set of all convex combinations of the vectors $\left\{e_{n}\right\}$, the convex closure of the function $f$ is an identical zero on the set $\mathcal{A}_{p}$. Therefore, $\overline{\mathrm{co}} f(0) \neq f(0)$. This example shows also that for the set $\mathcal{A}_{p}$ such that $\mathcal{A}_{p}=\overline{\mathrm{co}}\left(\operatorname{extr} \mathcal{A}_{p}\right)$ and map (2) is open (this follows from a theorem in [15] combined with the strict convexity of the space $l_{p}$ for $p>1$ and with the proof of theorem 1 in [9]) the assertion of corollary 2 in [9] does not hold, that is, a continuous function on the closed set extr $\mathcal{A}_{p}$ need not have a convex continuous (or even lower semicontinuous) extension to $\mathcal{A}_{p}$.

A Hilbert cube is the set $\mathcal{H}_{a}=\left\{x \in l_{2}|| x^{i} \mid \leq a^{i}, i \in \mathbb{N}\right\}$, where $a=\left\{a^{i}\right\}_{i \in \mathbb{N}}$ is an arbitrary sequence of positive numbers. Let us show that the following alternative holds for any Hilbert cube.

Proposition 16. If $\|a\|_{2}<+\infty$, then the set $\mathcal{H}_{a}$ is compact; otherwise, if $\|a\|_{2}=+\infty$, then it is not even pointwise $\mu$-compact. 
Proof. The first assertion is well known. It follows, for instance, from the compactness criterion for $l_{2}$. If $\|a\|_{2}=+\infty$, then we split the sequence $a$ into blocks so that each block contains finitely many consecutive elements the sum of whose squares exceeds 1 . Suppose that the $n$-th block consists of elements $a^{k}, a^{k+1}, \ldots, a^{k+m}$. We write $b_{n}=\sum_{i=k}^{k+m} a^{i} e_{i}$ for every $n$ and denote by $\mathcal{L}$ the closure of the linear span of the elements $b_{n}, n \in \mathbb{N}$. The set $\mathcal{L}$ is a Hilbert space with orthogonal basis $\left\{b_{n}\right\}$. The unit ball of the space $\mathcal{L}$ is contained in $\mathcal{H}_{a}$. Since a ball in a Hilbert space is not pointwise $\mu$-compact, it follows that the set $\mathcal{H}_{a}$ is not so either.

\section{The CE-properties of $\mu$-compact convex sets}

In the 1970s various properties of convex compact sets, in particular, the continuity properties of the convex hulls of continuous functions have been intensively studied. Vesterstrom proved in [10] the relation between continuity of the convex hull 4 of arbitrary continuous function and the openness of the barycenter map. He also conjectured the equivalence between continuity of the convex hull of any continuous concave function (called the CE-property in [11]) and the continuity of the convex hull of any continuous function (called the strong CE-property in [9]). This conjecture was approved by O'Brien [12, who showed the equivalence of these properties to the openness of the convex mixture map $(x, y, \lambda) \mapsto \lambda x+(1-\lambda) y$. In the subsequent papers the latter property was studied for convex sets that are not necessarily compact, and was called the stability property. Convex sets having this property were called stable convex sets [13]. The relations between the stability property and several other properties of convex sets were also revealed [14, [15].

In this section we generalize the Vesterstrom-O'Brien theory to the class of $\mu$-compact convex sets. The first partial result in this direction was obtained in [9], where the $\mu$-compact version of theorem 3.1 in [10] was proved. The following theorem is the $\mu$-compact generalization of the main result in [12].

Theorem 1. For a convex $\mu$-compact set $\mathcal{A}$ the following properties are equivalent:

(i) the map $\mathcal{A} \times \mathcal{A} \ni(x, y) \mapsto \frac{x+y}{2} \in \mathcal{A}$ is open (the stability property);

(ii) the $\operatorname{map} M(\mathcal{A}) \ni \mu \mapsto \mathbf{b}(\mu) \in \mathcal{A}$ is open;

(iii) the map $M(\overline{\operatorname{extr} \mathcal{A}}) \ni \mu \mapsto \mathbf{b}(\mu) \in \mathcal{A}$ is open,5

(iv) the convex hull of an arbitrary function in $C(\mathcal{A})$ is continuous (the strong CE-property);

(v) the convex hull of an arbitrary function in $Q(\mathcal{A})$ is continuous (the CE-property).

\footnotetext{
${ }^{4}$ By corollary I.3.6 in [2] the convex hull of any continuous function on a compact set coincides with the convex closure of this function.

${ }^{5}$ This map is surjective by Proposition 5 ,
} 
Equivalent properties (i) - (v) imply the closedness of the set $\operatorname{extr} \mathcal{A}$.

Remark 5. Property (i) in Theorem 1 is equivalent to the openness of the map $\mathcal{A} \times \mathcal{A} \times[0,1] \ni(x, y, \lambda) \mapsto \lambda x+(1-\lambda) y \in \mathcal{A}$ [14. Properties (iv) and (v) in Theorem 1 can be formulated as the continuity of the convex closure and its coincidence with the convex hull for any function in $C(\mathcal{A})$ and in $Q(\mathcal{A})$ respectively.

Remark 6. If properties (i) - (v) hold for $\mu$-compact set $\mathcal{A}$, then the family $F(\mathcal{A})$ in Proposition 2 can be chosen consisting of lower semicontinuous functions. Indeed, by using property (ii) it is easy to show that the functions $f_{\varphi}$ constructed in the proof of Proposition 2 are lower semicontinuous.

The proof of Theorem 1. Note first that (v) implies the closedness of the set extr $\mathcal{A}$ by Proposition 8 , since (v) guarantees the closedness of the set $\mathcal{B}_{f}=\{x \in \mathcal{A} \mid f(x)=\overline{\operatorname{co}} f(x)\}$ for any function $f \in Q(\mathcal{A})$.

$(\mathrm{v}) \Rightarrow$ (iii) The proof of this part of theorem (as well as the proof of the analogous part of theorem 3.2 in [10]) can be realized by means of lemma 2.1 in [10]. That lemma can be proved without the compactness assumption by the following observation: if $X$ is a compact space and $Y$ is an arbitrary topological space then the image of any closed subset of $X \times Y$ under the canonical projection $X \times Y \ni(x, y) \mapsto y \in Y$ is a closed subset of $Y$.

By representation (3) the convex closure of any function $f$ in $Q(\mathcal{A})$ is determined by the expression

$$
\overline{\mathrm{co}} f(x)=\inf _{\mu \in M_{x}(\operatorname{extr} \mathcal{A})} \mu(f), \quad \forall x \in \mathcal{A}, \quad \text { where } \mu(f)=\int_{\operatorname{extr} \mathcal{A}} f(x) \mu(d x) .
$$

Hence (v) yields the continuity and boundedness of the function

$$
\mathcal{A} \ni x \mapsto \sup \{\mu(f) \mid \mu \in M(\operatorname{extr} \mathcal{A}), b(\mu)=x\}
$$

for any function $f$ in $P(\mathcal{A})$. The above-mentioned generalization of lemma 2.1 in [10] with $K=M(\mathcal{A}), M=M(\operatorname{extr} \mathcal{A})$ and $K^{\prime}=\mathcal{A}$ implies the openness of the map

$$
M(\operatorname{extr} \mathcal{A}) \ni \mu \quad \mapsto \quad \mathbf{b}(\mu) \in \mathcal{A}
$$

provided the set $M(\operatorname{extr} \mathcal{A})$ is endowed with the topology, which has the prebase consisting of the sets $\{\mu \in M(\operatorname{extr} \mathcal{A}) \mid \mu(f)>0\}, f \in P(\mathcal{A})$. Following the terminology in [10], this topology will be called the $p$-topology. This is the weakest topology providing lower semicontinuity of the functionals $\mu \mapsto \mu(f)$ for any function $f \in P(\mathcal{A})$.

By using Lemma 6 (see Appendix) we will show that the openness of map (6) in the $p$-topology on $M(\operatorname{extr} \mathcal{A})$ and the closedness of the set extr $\mathcal{A}$ proved above imply the openness of map (6) in the weak topology on $M(\operatorname{extr} \mathcal{A})[6$ To realize this it suffices to show that for an arbitrary converging sequence $\left\{x_{n}\right\} \subset \mathcal{A}$ and for an arbitrary net $\left\{\mu_{\lambda}\right\}_{\lambda \in \Lambda} \subset M(\operatorname{extr} \mathcal{A})$ such that

$$
b\left(\left\{\mu_{\lambda}\right\}_{\lambda \in \Lambda}\right) \subseteq\left\{x_{n}\right\} \text { and } \exists p-\lim _{\lambda} \mu_{\lambda}=\mu_{0}
$$

\footnotetext{
${ }^{6}$ In the case of compact set $\mathcal{A}$ the coincidence of these topologies on $M(\operatorname{extr} \mathcal{A})$ is proved in [10] (lemma 3.4). In the case of $\mu$-compact set $\mathcal{A}$ we can not prove this coincidence.
} 
where $\mu_{0}$ is a measure in $M(\operatorname{extr} \mathcal{A})$ such that $\mathbf{b}\left(\mu_{0}\right)=\lim _{n \rightarrow+\infty} x_{n}$, there exists a subnet of the net $\left\{\mu_{\lambda}\right\}_{\lambda \in \Lambda}$ that converges weakly to the measure $\mu_{0}$.

Let $\left\{x_{n}\right\}$ and $\left\{\mu_{\lambda}\right\}_{\lambda \in \Lambda}$ be the above sequence and net, respectively. Since the sequence is relatively compact, $\mu$-compactness of the set $\mathcal{A}$ and the inclusion $\mathbf{b}\left(\left\{\mu_{\lambda}\right\}_{\lambda \in \Lambda}\right) \subseteq\left\{x_{n}\right\}$ imply relative compactness of the net $\left\{\mu_{\lambda}\right\}_{\lambda \in \Lambda}$ in the weak topology and hence the existence of the subnet $\left\{\mu_{\lambda_{\pi}}\right\}_{\pi \in \Pi}$ weakly converging to a some measure $\nu \in M(\operatorname{extr} \mathcal{A})$. By the definitions of the weak topology and of the $p$-topology we have

$$
\nu(f)=\lim _{\pi} \mu_{\lambda_{\pi}}(f) \geq p-\liminf _{\lambda} \mu_{\lambda}(f) \geq \mu_{0}(f), \quad \forall f \in P(\mathcal{A}) .
$$

This means that $\nu \succ \mu_{0}$ (in the Choquet ordering). Closedness of the set extr $\mathcal{A}$ implies the maximality in $M(\mathcal{A})$ of any measure in $M(\operatorname{extr} \mathcal{A})$. This can be proved by using Theorem 2.2 in [5] and the arguments from the proof of Theorem 1.1 in [7], but it can also be immediately shown by using property (v) and coincidence of any function in $Q(\mathcal{A})$ with its convex hull on the set extr $\mathcal{A}$. Thus $\mu_{0}$ is the maximal measure in $M(\mathcal{A})$ and hence $\nu=\mu_{0}$.

(iii) $\Rightarrow$ (i) This implication follows from Proposition 5 and Proposition 17 below (with $X=\overline{\operatorname{extr} \mathcal{A}})$.

By Remark 5 the equivalence of properties (i), (ii) and (iv) for convex $\mu$-compact subsets of a Banach space is proved in [9, theorem 1]. That proof is easily extended to the class of sets considered in this paper.

The implication (iv) $\Rightarrow(\mathrm{v})$ is obvious.

In the proof of Theorem 1 we have involved the following result of the measure theory ([24, theorem 2.4]).

Proposition 17. Let $X$ be a complete separable metric space. The map

$$
M(X) \times M(X) \ni(\mu, \nu) \mapsto \frac{1}{2}(\mu+\nu) \in M(X)
$$

is open.

Since the set $M(X)$ is $\mu$-compact (Corollary 4), we arrive at the following observation.

Corollary 5. Properties (i) - (v) in Theorem 1 hold for the set of Borel probability measures on a complete separable metric space endowed with the weak convergence topology.

The $\mu$-compactness condition is essential in the proof of Theorem 1, it can not be removed without changing the whole structure of the proof. This motivates the conjecture that the class of $\mu$-compact convex sets is the maximal class of convex metrizable sets for which the Vesterstrom - O'Brien theory can be generalized. This conjecture can be justified by the following example, showing that even pointwise $\mu$-compactness is not sufficient for the proof of Theorem 1 .

Proposition 18. For any $p>1$ the pointwise $\mu$-compact simplex

$$
\Delta_{p}=\left\{x \in l_{p} \mid x \geq 0, \sum_{i=1}^{+\infty} x^{i} \leq 1\right\}
$$


in $l_{p}$ is stable, that is, it possesses property (i) in Theorem 1, which implies (ii), but it does not possess properties (iii) - (v).

Note that for $p=1$ the $\mu$-compact simplex $\Delta_{1}=\mathcal{A}_{1}$ has properties (i) $-(\mathrm{v})$ in Theorem 1 .

Proof. Example 1 shows that the simplex $\Delta_{p}$ does not possess properties (iv) and (v). Let us show that it does not possess (iii) either. Note that extr $\Delta_{p}=\left\{0, e_{i}, i \in \mathbb{N}\right\}$ and that the set $\Delta_{p}$ is a real simplex: for any its point $x$ there exists the unique measure on extr $\Delta_{p}$ with the barycenter $x$. The sequence of points $x_{n}=\left(\frac{1}{n}, \cdots \frac{1}{n}, 0, \cdots\right) \in \Delta_{p}$ (the first $n$ coordinates equal to $\frac{1}{n}$, the all others are zeros) converges to zero in $l_{p}$ as $n \rightarrow+\infty$, but it is easy to see that the corresponding sequence of measures on extr $\Delta_{p}$ does not converge to the single atom measure supported at the point 0 .

Let us now show that for an arbitrary $p>1$ the set $\Delta_{p}$ is stable, that is, it has property (i), which implies (ii) (see the proof of theorem 1 in [9]). It suffices to prove that for arbitrary points $a, b \subset \Delta_{p}, c=\frac{1}{2}(a+b)$ and for arbitrary $\varepsilon>0$, there exists $\delta>0$ with the following property: for any $z \in \Delta_{p}$ such that $\|z-c\|_{p}<\delta$ there exists a segment $[x, y] \subset \Delta_{p}$ centered at $z$, for which $\|x-a\|_{p}<\varepsilon$ and $\|y-b\|_{p}<\varepsilon$. By taking sufficiently small $\varepsilon$ it can be assumed that $\|a\|_{p}<1-\varepsilon$ and that $\|b\|_{p}<1-\varepsilon$. Otherwise the points $a$ and $b$ can be replaced by sufficiently close points belonging to the interior of $[a, b]$. Since the $l_{p}$-norm is strictly convex, the norms of $a$ and of $b$ become less then 1 . Then we choose a large $N$ so that for each of the points $a$ and $b$ the norm of the "tail" from the $(N+1)$ th coordinate is less than $\frac{1}{6} \varepsilon$, that is, $\left(\sum_{k=N+1}^{+\infty}\left(a^{k}\right)^{p}\right)^{1 / p}<\frac{1}{6} \varepsilon$ and $\left(\sum_{k=N+1}^{+\infty}\left(b^{k}\right)^{p}\right)^{1 / p}<\frac{1}{6} \varepsilon$. Consider the space $\mathbb{R}^{N}$, generated by the first $N$ coordinates. Denote by $\tilde{\Delta}_{p}$ and $\tilde{s}$ the restrictions of the set $\Delta_{p}$ and of arbitrary element $s \in l_{p}$ to this space. Since $\tilde{\Delta}_{p}$ is a simplex in $\mathbb{R}^{N}$ it is stable (see [13]) and hence one can take $\delta>0$ such that there exist points $\tilde{x}, \tilde{y} \in \tilde{\Delta}_{p}$, for which $\frac{1}{2}(\tilde{x}+\tilde{y})=\tilde{z},\|\tilde{x}-\tilde{a}\|_{p}<\frac{1}{3} \varepsilon$ and $\|\tilde{y}-\tilde{b}\|_{p}<\frac{1}{3} \varepsilon$, if $\|\tilde{z}-\tilde{c}\|_{p}<\delta$. Now for given $z \in \Delta_{p}$ and for arbitrary $t \in[-1,1]$ we define the points $x(t), y(t) \in l_{p}$ as follows:

$$
x^{k}(t)=\left\{\begin{array}{ll}
\tilde{x}^{k}, & k \leq N \\
(1+t) z^{k}, & k>N
\end{array} \quad y^{k}(t)=\left\{\begin{array}{cl}
\tilde{y}^{k}, & k \leq N \\
(1-t) z^{k}, & k>N
\end{array}\right.\right.
$$

By construction $\frac{1}{2}(x(t)+y(t))=z$ for any $t$, while the norms of the elements $x(t)$ and $y(t)$ do not exceed $1-\frac{1}{3} \varepsilon+2 \delta$. Indeed,

$$
\|\tilde{x}\|_{p} \leq\|\tilde{a}\|_{p}+\|\tilde{x}-\tilde{a}\|_{p} \leq 1-\varepsilon+\frac{1}{3} \varepsilon=1-\frac{2}{3} \varepsilon,
$$

while the norm of the "tail" of $(1+t) z$ does not exceed the sum of norms of the "tails" of $2 c$ and $2(z-c)$, that is, it does not exceed $2\left(\frac{1}{6} \varepsilon+\delta\right)$. Taking the sum we obtain $\|x(t)\|_{p} \leq 1-\frac{1}{3} \varepsilon+2 \delta$, and the same is true for $y(t)$. For $\delta \leq \frac{1}{6} \varepsilon$ we get $\|x(t)\|_{p} \leq 1$ and $\|y(t)\|_{p} \leq 1$. Let us show that there exists $\tau \in[-1,1]$, for which $\|x(\tau)\|_{1} \leq 1$ and $\|y(\tau)\|_{1} \leq 1$, and whence $x(\tau), y(\tau) \in \Delta_{p}$. It is clear that $\|\tilde{x}\|_{1} \leq 1$ and $\|\tilde{y}\|_{1} \leq 1$. For the sake of being defined, suppose $\|\tilde{x}\|_{1} \geq\|\tilde{y}\|_{1}$. If $\|y(-1)\|_{1} \leq 1$, then one can take 
$t=-1$, since $\|x(-1)\|_{1}=\|\tilde{x}\|_{1} \leq 1$. If $\|y(-1)\|_{1}>1$, then $\|y(-1)\|_{1}>\|x(-1)\|_{1}$, and since $\|y(1)\|_{1} \leq\|x(1)\|_{1}$, applying the continuity argument, we conclude that there is $\tau \in[-1,1]$ such that $\|y(\tau)\|_{1}=\|x(\tau)\|_{1}$. Since $\frac{1}{2}(x(\tau)+y(\tau))=z$, we have $\|x(\tau)\|_{1}=\|y(\tau)\|_{1}=\|z\|_{1} \leq 1$.

Finally, the norm of the difference $\|x(\tau)-a\|_{p}$ does not exceed $\frac{1}{3} \varepsilon$ for the first $N$ coordinates, while for the other coordinates it does not exceed the maximal norm of the two "tails": of the element $a$ and of $2 z$. Hence,

$$
\|x(\tau)-a\|_{p} \leq \frac{1}{3} \varepsilon+\max \left\{\frac{1}{6} \varepsilon, 2\left(\frac{1}{6} \varepsilon+\delta\right)\right\}=\frac{2}{3} \varepsilon+2 \delta .
$$

For $\delta<\frac{1}{6} \varepsilon$ we obtain $\|x(\tau)-a\|_{p}<\varepsilon$, and similarly $\|y(\tau)-b\|_{p}<\varepsilon$. By setting $x=x(\tau), y=y(\tau)$, we complete the proof.

\section{Applications to quantum information theory}

An important example of convex $\mu$-compact sets, for which the equivalent properties of Theorem 1 hold, is the set $\mathfrak{S}(\mathcal{H})$ of quantum states 7 The quantum states are density operators (positive operators with trace equal to 1 ) in a separable Hilbert space $\mathcal{H}$ [27]. The set of extreme points of the set $\mathfrak{S}(\mathcal{H})$ consists of one-dimensional projectors called pure states. The $\mu$-compactness and the stability property (i) in Theorem 1 of the set $\mathfrak{S}(\mathcal{H})$ are established in [22, proposition 2] and in [25, lemma 3] respectively. These properties are used essentially in the study of characteristics of quantum states and of quantum channels. For instance, $\mu$-compactness of the set $\mathfrak{S}(\mathcal{H})$ makes it possible to prove that any nonentangled state (see below) of composite quantum system can be represented as an average (barycenter) state of some generalized ensemble of pure product states (probability measure on the set of product pure states) [26]. The stability property of the set $\mathfrak{S}(\mathcal{H})$ plays the crucial role in the proof of the lower semicontinuity property of the $\chi$-function of an arbitrary quantum channel, which is an important characteristic related to the classical capacity of this channel [25].

In this section we consider a result following directly from the generalized VesterstromO'Brien theorem (Theorem 1) applied to the stable set $\mathfrak{S}(\mathcal{H})$.

According to the quantum mechanical formalism, states of composite quantum system, arising as a result of joining two quantum systems represented by two Hilbert spaces $\mathcal{H}$ and $\mathcal{K}$, correspond to the density operators in the tensor product $\mathcal{H} \otimes \mathcal{K}$ of these spaces. A specific property of the quantum mechanical statistical model (in comparison to the classical one) is the existence of the so called entangled states of composite system, which can not be represented as convex combinations of product states describing independent subsystems. Entanglement can be considered as a special purely quantum correlation, which is the base for the construction of different quantum algorithms, quantum criptographical protocols and

\footnotetext{
${ }^{7}$ The set $\mathfrak{S}(\mathcal{H})$ is compact if and only if $\operatorname{dim} \mathcal{H}<+\infty$.
} 
systems of information transmissions, which attract a lot of attention of scientists in the last two decades (see [27], chapter 3). That is why the study of the entanglement, in particular, of its quantitative characteristics, is one of the main problems of the quantum information theory.

Let $\mathcal{H}$ and $\mathcal{K}$ be separable Hilbert spaces. A state $\omega \in \mathfrak{S}(\mathcal{H} \otimes \mathcal{K})$ is called nonentangled if it belongs to the convex closure of the set of product states, that is, of states of the form $\rho \otimes \sigma$, where $\rho \in \mathfrak{S}(\mathcal{H})$ and $\sigma \in \mathfrak{S}(\mathcal{K})$; otherwise it is called entangled.

Entanglement monotone is an arbitrary function $E$ on the set $\mathfrak{S}(\mathcal{H} \otimes \mathcal{K})$ that possesses the following properties (see [28], 29]):

$\mathrm{E}-1)\{E(\omega)=0\} \Leftrightarrow\{$ the state $\omega$ is nonentangled $\}$

E-2) Monotonicity under Local Operations and Classical Communications (LOCC), which means

$$
E(\omega) \geq \sum_{i} \pi_{i} E\left(\omega_{i}\right)
$$

for arbitrary state $\omega \in \mathfrak{S}(\mathcal{H} \otimes \mathcal{K})$ and arbitrary LOCC-operation, transforming the state $\omega$ into the set $\left\{\omega_{i}\right\}$ of states with the probability distribution $\left\{\pi_{i}\right\}$ (see details in [29]).

E-3) Convexity of the function $E$ on the set $\mathfrak{S}(\mathcal{H} \otimes \mathcal{K})$, which means

$$
E\left(\sum_{i} \pi_{i} \omega_{i}\right) \leq \sum_{i} \pi_{i} E\left(\omega_{i}\right)
$$

for arbitrary finite set $\left\{\omega_{i}\right\}$ of states in $\mathfrak{S}(\mathcal{H} \otimes \mathcal{K})$ and probability distribution $\left\{\pi_{i}\right\}$.

The standard method of "generation" of entanglement monotones (EM) in the case of finite dimensional spaces $\mathcal{H}$ and $\mathcal{K}$ is the convex roof construction (see [29, [30]). In accordance with this method, for an arbitrary concave continuous nonnegative function $f$ on the set $\mathfrak{S}(\mathcal{H})$ such that

$$
f^{-1}(0)=\operatorname{extr} \mathfrak{S}(\mathcal{H}) \text { and } \quad f(\rho)=f\left(U \rho U^{*}\right)
$$

for any state $\rho$ in $\mathfrak{S}(\mathcal{H})$ and any unitary operator $U$ in the space $\mathcal{H}$ the corresponding EM $E^{f}$ is defined by

$$
E^{f}(\omega)=\inf _{\left\{\pi_{i}, \omega_{i}\right\} \in M_{\omega}(\operatorname{extr} \mathfrak{S}(\mathcal{H} \otimes \mathcal{K}))} \sum_{i} \pi_{i} f \circ \Theta\left(\omega_{i}\right), \quad \omega \in \mathfrak{S}(\mathcal{H} \otimes \mathcal{K}),
$$

where $\Theta: \omega \mapsto \operatorname{Tr}_{\mathcal{K}} \omega$ is the partial trace [27] (by the spectral theorem the right hand side of (9) is well defined). If the von Neumann entropy $H(\rho)=-\operatorname{Tr} \rho \log \rho$ is used as a function $f$, then this method provides the construction of the Entanglement of Formation, which is one of the most useful entanglement measures 8 [29].

In what follows the properties of the function $E^{f}$ defined by (9) in case of infinite dimensional spaces $\mathcal{H}$ and $\mathcal{K}$ are considered.

An important problem in constructing of EM is to analyse the continuity properties, in particular, to prove its continuity on the entire state space $\mathfrak{S}(\mathcal{H} \otimes \mathcal{K}$ ) (formally the last

\footnotetext{
${ }^{8}$ Entanglement measure is a EM having the particular additional properties [29].
} 
property is not included in the definition of EM, but in finite dimensions it is considered as a natural requirement). Note that the continuity of the function $E^{f}$ is not obvious even in the finite dimensional case and in general it is proved by using the explicit form of the function $f$. By Theorem 1 the $\mu$-compactness and stability of the set $\mathfrak{S}(\mathcal{H} \otimes \mathcal{K})$ guarantee continuity of the function $E^{f}$ on this set for an arbitrary continuous function $f$ in the both finite and infinite dimensional cases.

Theorem 2. Let $f$ be a concave continuous nonnegative function on the set $\mathfrak{S}(\mathcal{H})$ satisfying condition (8). Then the function $E^{f}$ defined by (9) is an entanglement monotone that is continuous on the set $\mathfrak{S}(\mathcal{H} \otimes \mathcal{K})$.

Proof. Nonnegativity, concavity, and continuity of the function $f$ imply its boundedness. By Theorem 1 the stability property of the $\mu$-compact set $\mathfrak{S}(\mathcal{H} \otimes \mathcal{K})$ guarantees the continuity of the function $\operatorname{co}(f \circ \Theta)$ and therefore, its coincidence with the function $\overline{\mathrm{co}}(f \circ \Theta)$, which by Proposition 6 has the following representation

$$
\overline{\mathrm{co}}(f \circ \Theta)(\omega)=\inf _{\mu \in M_{\omega}(\mathfrak{S}(\mathcal{H} \otimes \mathcal{K}))} \int_{\mathfrak{S}(\mathcal{H} \otimes \mathcal{K})}(f \circ \Theta)(\varpi) \mu(d \varpi), \quad \omega \in \mathfrak{S}(\mathcal{H} \otimes \mathcal{K}),
$$

where the infimum is achieved at some particular measure $\mu_{\omega}$ in $M_{\omega}(\mathfrak{S}(\mathcal{H} \otimes \mathcal{K}))$. By concavity, continuity, and boundedness of the function $f \circ \Theta$ one can assume that $\mu_{\omega}$ is a measure in $M_{\omega}(\operatorname{extr} \mathfrak{S}(\mathcal{H} \otimes \mathcal{K}))$. Hence the definition of the function $E^{f}$ and concavity of the function $f \circ \Theta$ imply $E^{f}=\operatorname{co}(f \circ \Theta)=\overline{\mathrm{co}}(f \circ \Theta)$.

By (8) the nonnegative function $f \circ \Theta$ vanishes on a pure state in $\mathfrak{S}(\mathcal{H} \otimes \mathcal{K})$ if and only if this state is product. As shown in [26], a state $\omega$ is nonentangled if and only if there exists such measure $\mu_{\omega}$ supported by pure product states that $\mathbf{b}\left(\mu_{\omega}\right)=\omega$. Thus the above remark shows that condition E-1 is fulfilled for the function $E^{f}=\overline{\mathrm{co}}(f \circ \Theta)$.

Condition E-2 for the function $E^{f}$ is established in the same way as in the finite dimensional case (see [29]).

Condition E-3 for the function $E^{f}$ follows from its definition.

Example 2. Generalizing the observation in [30] to the infinite dimensional case, consider the family of continuous concave functions

$$
f_{\alpha}(\rho)=2\left(1-\operatorname{Tr} \rho^{\alpha}\right), \quad \alpha>1,
$$

on the set $\mathfrak{S}(\mathcal{H})$ with $\operatorname{dim} \mathcal{H} \leq+\infty$. It is easy to see that all the functions in this family satisfy assumptions (8). By Theorem $2\left\{E^{f_{\alpha}}\right\}_{\alpha>1}$ is a family of entanglement monotones, which are continuous and bounded on the set $\mathfrak{S}(\mathcal{H} \otimes \mathcal{K})$ with $\operatorname{dim} \mathcal{H} \leq+\infty$ and $\operatorname{dim} \mathcal{K} \leq+\infty$. The case $\alpha=2$ is of special interest since the entanglement monotone $E^{f_{2}}$ can be considered as an infinite dimensional generalization of the notion of I-tangle [31.

\section{Possible generalizations and open questions}

Proposition 1 actually gives an equivalent definition of the $\mu$-compactness property for convex sets of the class considered in this paper. A convex set $\mathcal{A}$ is $\mu$-compact if and only if for 
arbitrary compact set $\mathcal{K} \subseteq \mathcal{A}$ and for arbitrary $\varepsilon>0$ there exists a compact set $\mathcal{K}_{\varepsilon} \subseteq \mathcal{A}$ such that for any expansion of a point $x \in \mathcal{K}$ into convex combination of points in $\mathcal{A}$ the total weight of points belonging to the set $\mathcal{K}_{\varepsilon}$ is not less than $1-\varepsilon$. This property of (arbitrary!) convex set can be called generalized $\mu$-compactness, or, in short, $\tilde{\mu}$-compactness. By Proposition 1 the $\tilde{\mu}$-compactness property means $\mu$-compactness for convex bounded subsets of locally convex spaces, that are complete separable metric spaces. The above definition of $\tilde{\mu}$-compactness is translated without any change to any convex closed subsets of linear topological spaces, not necessarily bounded. The definition of $\mu$-compactness is, in contrast, not generalized to unbounded sets, since for unbounded sets the barycenter map may not be well defined: the integral $\mathbf{b}(\mu)=\int_{\mathcal{A}} x \mu(d x)$ may not exist for some measures $\mu \in M(\mathcal{A})$. Thus the notion of $\tilde{\mu}$-compactness generalizes the notion of $\mu$-compactness to a wider class of convex sets. Similar to the case of $\mu$-compact sets, the intersection and the Cartesian product of finite or countable family of $\tilde{\mu}$-compact sets is $\tilde{\mu}$-compact, the convex closed subset of a $\tilde{\mu}$-compact set is $\tilde{\mu}$-compact. The proof of this assertions is literally the same as the proof of Proposition 4. A complete analog of Proposition 3 for continuous transformations of $\mu$-compact sets holds as well. Nontrivial examples of $\tilde{\mu}$-compacts sets appear even in the finite dimensional case.

Lemma 3. An arbitrary convex closed pointed (not containing any line) cone in $\mathbb{R}^{d}$ is $\tilde{\mu}$-compact.

Proof. Let $\mathcal{C} \subset \mathbb{R}^{d}$ be a convex pointed cone. Hence there exists a vector $a \in \mathbb{R}^{d}$ such that $\inf _{x \in \mathcal{C},\|x\|=1}(x, a)>0$ [32, p.53]. Then for each $r>0$ the truncated cone

$$
\mathcal{C}_{r}=\{x \in \mathcal{C},(x, a) \leq r\}
$$

is compact. Arbitrary compact set $\mathcal{K} \subset \mathcal{C}$ can be embedded into a particular truncated cone $\mathcal{C}_{r}$. Then for each $\varepsilon>0$ the compact set $\mathcal{K}_{\varepsilon}=\mathcal{C}_{\frac{r}{\varepsilon}}$ has the required property.

The following result from the convex geometry is well known, so we omit its proof.

Lemma 4. The following properties of a convex closed set $\mathcal{A} \subset \mathbb{R}^{d}$ are equivalent:

(i) $\mathcal{A}$ is contained in a convex pointed cone;

(ii) $\mathcal{A}$ has at least one extreme point;

(iii) $\mathcal{A}$ does not contain a straight line;

(iv) the polar of the set $\mathcal{A}$ has a nonempty interior.

By applying Lemma 3 we conclude that property (i) implies $\tilde{\mu}$-compactness of the set $\mathcal{A}$. On the other hand, a line is not $\tilde{\mu}$-compact, hence $\tilde{\mu}$-compactness implies property (iii). Thus, we obtain the following result.

Proposition 19. In the space $\mathbb{R}^{d}$ the $\tilde{\mu}$-compactness is equivalent to each of properties (i) - (iv) in Lemma 4. 
Positive cones in the spaces $l_{p}$ and $L_{p}(X)$ are $\tilde{\mu}$-compact in the weak topology. The proof is analogous to the proof of Lemma 3 and is based on the weak compactness of bounded sets in this spaces. The positive cone in $l_{1}$ (Proposition 9), the cone of finite Borel measures on a complete separable metric space (Proposition 10), and the cone of positive operators in the Shatten class of order $p=1$ (Proposition 11) are $\tilde{\mu}$-compact and, in contrast to these propositions, one need not take bounded parts of these cones. Thus, $\tilde{\mu}$-compactness substantially extends the notion of $\mu$-compactness. This motivates our first question.

Question 1. To what extent are the results of this paper generalized to $\tilde{\mu}$-compact sets?

The following questions concern $\mu$-compact sets.

Question 2. Do there exist $\mu$-compact noncompact sets in the spaces $L_{p}$ and $l_{p}$ with $p>1$ ?

Question 3. Consider a Banach lattice. Under what conditions is the bounded part of the positive cone in it $\mu$-compact?

Question 4. Under what conditions on a convex set $\mathcal{A}$, is the convex hull of an arbitrary continuous bounded function on this set continuous?

The latter property holds for stable $\mu$-compact sets (Theorem 1), but it does not hold for stable pointwise $\mu$-compact sets that are not $\mu$-compact (Proposition 7). The unit ball in the space $l_{2}$ possesses this property (Remark 3), but the positive part of this ball does not (Example 1).

\section{Appendix}

\subsection{The compactness criterion for subsets of the cone $\mathfrak{L}_{+}\left(\mathcal{H}, \mathcal{H}^{\prime}\right)$}

Let $\mathfrak{L}_{+}\left(\mathcal{H}, \mathcal{H}^{\prime}\right)$ be the cone of linear continuous positive maps from the Banach space $\mathfrak{T}(\mathcal{H})$ of trace-class operators in a separable Hilbert space $\mathcal{H}$ into the similar Banach space $\mathfrak{T}\left(\mathcal{H}^{\prime}\right)$. The compactness criterion for subsets of this cone in the strong operator topology is presented in the following lemma.

Lemma 5. 1) A closed bounded subset $\mathfrak{L}_{0} \subseteq \mathfrak{L}_{+}\left(\mathcal{H}, \mathcal{H}^{\prime}\right)$ is compact in the strong operator topology if in $\mathfrak{S}(\mathcal{H})$ there exists such full rank state $\sigma$ that $\{\Phi(\sigma)\}_{\Phi \in \mathfrak{L}_{0}}$ is a compact subset of $\mathfrak{T}\left(\mathcal{H}^{\prime}\right)$.

2) If a subset $\mathfrak{L}_{0} \subseteq \mathfrak{L}_{+}\left(\mathcal{H}, \mathcal{H}^{\prime}\right)$ is compact in the strong operator topology then $\{\Phi(\sigma)\}_{\Phi \in \mathfrak{L}_{0}}$ is a compact subset of $\mathfrak{T}\left(\mathcal{H}^{\prime}\right)$ for any state $\sigma$ in $\mathfrak{S}(\mathcal{H})$.

Proof. 1) Let $\{|i\rangle\}$ be the basis of eigenvectors of the state $\sigma$ arranged in nonincreasing order and $\mathcal{H}_{m}$ be the eigen subspace generated by the first $m$ vectors of this basis.

Let $\left\{\Phi_{n}\right\}$ be an arbitrary sequence of maps in $\mathfrak{L}_{0}$.

Show that for each $m$ for an arbitrary operator $A$ in $\mathfrak{T}\left(\mathcal{H}_{m}\right)$ there exists such subsequence $\left\{\Phi_{n_{k}}\right\}$ that the sequence $\left\{\Phi_{n_{k}}(A)\right\}_{k}$ converges in $\mathfrak{T}\left(\mathcal{H}^{\prime}\right)$. Suppose first that $A \geq 0$. Since $A \in \mathfrak{T}\left(\mathcal{H}_{m}\right)$ there exists such $\lambda_{A}>0$ that $\lambda_{A} A \leq \sigma$. By the compactness criterion for subsets of $\mathfrak{T}\left(\mathcal{H}^{\prime}\right)$ (see the Appendix in [21]) for arbitrary $\varepsilon>0$ there exists finite rank projector 
$P_{\varepsilon} \in \mathfrak{B}\left(\mathcal{H}^{\prime}\right)$ such that $\operatorname{Tr}\left(I_{\mathcal{H}^{\prime}}-P_{\varepsilon}\right) \Phi(\sigma)<\varepsilon$, and hence $\operatorname{Tr}\left(I_{\mathcal{H}^{\prime}}-P_{\varepsilon}\right) \Phi(A)<\lambda_{A}^{-1} \varepsilon$ for all $\Phi \in \mathfrak{L}_{0}$. By the same compactness criterion the set $\{\Phi(A)\}_{\Phi \in \mathfrak{L}_{0}}$ is compact. This implies existence of the desired subsequence for a positive operator $A$. Existence of such subsequence for an arbitrary operator $A \in \mathfrak{T}\left(\mathcal{H}_{m}\right)$ follows from representation of this operator as a linear combination of positive operators in $\mathfrak{T}\left(\mathcal{H}_{m}\right)$.

Thus for each $m$ an arbitrary sequence $\left\{\Phi_{n}\right\} \subset \mathfrak{L}_{0}$ contains such subsequence $\left\{\Phi_{n_{k}}\right\}$ that there exists

$$
\lim _{k \rightarrow+\infty} \Phi_{n_{k}}(|i\rangle\langle j|)=C_{i j}^{m}
$$

for all $i, j=\overline{1, m}$, where $\left\{C_{i j}^{m}\right\}$ are particular operators in $\mathfrak{T}\left(\mathcal{H}^{\prime}\right)$.

For arbitrary $m^{\prime}>m$, by applying the above observation to the sequence $\left\{\Phi_{n_{k}}\right\}_{k}$, we obtain such subsequence of the sequence $\left\{\Phi_{n}\right\}$ that (11) holds for all $i, j=\overline{1, m^{\prime}}$ with such set of operators $\left\{C_{i j}^{m^{\prime}}\right\}$, that $C_{i j}^{m^{\prime}}=C_{i j}^{m}$ for all $i, j=\overline{1, m}$.

By using this construction one can show existence of the set $\left\{C_{i j}\right\}_{i, j=1}^{+\infty}$ of operators having the following property: for each $m$ there exists such subsequence $\left\{\Phi_{n_{k}}\right\}$ of the sequence $\left\{\Phi_{n}\right\}$ that (11) holds with $C_{i j}^{m}=C_{i j}$ for all $i, j=\overline{1, m}$.

Consider the map $\Phi_{*}$ defined on the set $\bigcup_{m \in \mathbb{N}} \mathfrak{T}\left(\mathcal{H}_{m}\right)$ as follows

$$
\Phi_{*}: \sum_{i, j} a_{i j}|i\rangle\langle j| \mapsto \sum_{i, j} a_{i j} C_{i j} \in \mathfrak{T}\left(\mathcal{H}^{\prime}\right) .
$$

This map is linear by construction. It is easy to prove its positivity and boundedness. Indeed, by the property of the set $\left\{C_{i j}\right\}$ for an arbitrary operator $A \in \bigcup_{m} \mathfrak{T}\left(\mathcal{H}_{m}\right)$ there exists a subsequence $\left\{\Phi_{n_{k}}\right\}$ of the sequence $\left\{\Phi_{n}\right\}$ such that $\Phi_{*}(A)=\lim _{k \rightarrow+\infty} \Phi_{n_{k}}(A)$. Thus positivity and boundedness of the map $\Phi_{*}$ follows from positivity of the maps in the sequence $\left\{\Phi_{n}\right\}$ and uniform boundedness of these maps. Since the set $\bigcup_{m} \mathfrak{T}\left(\mathcal{H}_{m}\right)$ is dense in $\mathfrak{T}(\mathcal{H})$, the map $\Phi_{*}$ can be extended to a linear positive bounded map from $\mathfrak{T}(\mathcal{H})$ into $\mathfrak{T}\left(\mathcal{H}^{\prime}\right)$ (denoted by the same symbol $\Phi_{*}$ ).

Show that the map $\Phi_{*}$ is a limit point of the sequence $\left\{\Phi_{n}\right\}$ in the strong operator topology. This topology on bounded subsets of $\mathfrak{L}_{+}\left(\mathcal{H}, \mathcal{H}^{\prime}\right)$ can be determined by countable family of seminorms $\Phi \mapsto\left\|\Phi\left(\rho_{i}\right)\right\|_{1}$, where $\left\{\rho_{i}\right\}$ is an arbitrary countable dense subset of the set $\mathfrak{S}(\mathcal{H}) \cdot \sqrt{9}$ We choose the set of states in $\bigcup_{m} \mathfrak{T}\left(\mathcal{H}_{m}\right)$ on the role of this subset. An arbitrary vicinity of the map $\Phi_{*}$ contains vicinity of the form

$$
\left\{\Phi \in \mathfrak{L}\left(\mathcal{H}, \mathcal{H}^{\prime}\right) \mid\left\|\left(\Phi-\Phi_{*}\right)\left(\rho_{i_{t}}\right)\right\|_{1}<\varepsilon, t=\overline{1, p}\right\},
$$

where $\left\{\rho_{i_{t}}\right\}_{t=1}^{p}$ is a finite subset of the above set of states and $\varepsilon>0$. Since $\left\{\rho_{i_{t}}\right\}_{t=1}^{p} \subset \mathfrak{T}\left(\mathcal{H}_{m}\right)$ for a particular $m$, the construction of the map $\Phi_{*}$ implies existence of such subsequence $\left\{\Phi_{n_{k}}\right\}$ of the sequence $\left\{\Phi_{n}\right\}$ that $\Phi_{*}\left(\rho_{i_{t}}\right)=\lim _{k \rightarrow+\infty} \Phi_{n_{k}}\left(\rho_{i_{t}}\right)$ for all $t=\overline{1, p}$. Hence at least one element of the sequence $\left\{\Phi_{n}\right\}$ is contained in the above vicinity.

Thus the map $\Phi_{*}$ is a limit point of the sequence $\left\{\Phi_{n}\right\}$ in the strong operator topology. By metrizability of the strong operator topology on bounded subsets of the cone $\mathfrak{L}_{+}\left(\mathcal{H}, \mathcal{H}^{\prime}\right)$

\footnotetext{
${ }^{9}$ Here the possibility to express an arbitrary operator in $\mathfrak{T}(\mathcal{H})$ as a linear combination of four states in $\mathfrak{S}(\mathcal{H})$ is used.
} 
this implies existence of a subsequence of the sequence $\left\{\Phi_{n}\right\}$ converging to the map $\Phi_{*}$. Compactness of the set $\mathfrak{L}_{0}$ is proved.

2) This assertion immediately follows from the definition of the strong operator topology.

\subsection{The openness criterion}

Lemma 6. Let $\varphi$ be a map from a topological space $X$ to a metric space $Y$. The following assertions are equivalent:

(i) the map $\varphi$ is open;

(ii) for arbitrary $x_{0} \in X$ and arbitrary sequence $\left\{y_{n}\right\} \subset Y$, converging to $y_{0}=\varphi\left(x_{0}\right)$, there exists a subnet $\left\{y_{n_{\lambda}}\right\}_{\lambda \in \Lambda}$ of the sequence $\left\{y_{n}\right\}$ and a net $\left\{x_{\lambda}\right\}_{\lambda \in \Lambda}$, converging to $x_{0}$, such that $\varphi\left(x_{\lambda}\right)=y_{n_{\lambda}}$ for all $\lambda \in \Lambda$.

Proof. (i) $\Rightarrow$ (ii) Let $\mathfrak{U}$ be the set of all vicinities of the point $x_{0}$. Then the set $\Lambda$ of all pairs $\lambda=(U, k)$, where $U \in \mathfrak{U}$ and $k \in \mathbb{N}$, with the partial order

$$
\left\{\lambda_{1}=\left(U_{1}, k_{1}\right) \succ \lambda_{2}=\left(U_{2}, k_{2}\right)\right\} \Leftrightarrow\left\{k_{1} \geq k_{2} \text { and } U_{1} \subseteq U_{2}\right\}
$$

is directed. For each $\lambda=(U, k)$ the set $W_{\lambda}=\varphi(U) \cap V_{k}$, where $V_{k}$ is the open ball in $Y$ with the center $y_{0}$ and radius $1 / k$, is a vicinity of the point $y_{0}$. Hence there exists minimal natural $n_{\lambda}$ such that $y_{n_{\lambda}} \in W_{\lambda}$. It is easy to see that $\left\{y_{n_{\lambda}}\right\}_{\lambda \in \Lambda}$ is a subnet of the sequence $\left\{y_{n}\right\}$. For each $\lambda=(U, k)$ there exists such $x_{\lambda} \in U$ that $\varphi\left(x_{\lambda}\right)=y_{n_{\lambda}}$. It is clear that the net $\left\{x_{\lambda}\right\}_{\lambda \in \Lambda}$ converges to $x_{0}$.

(ii) $\Rightarrow$ (i) If there exists such open set $U \subseteq X$ that the set $\varphi(U)$ is not open then there exist $y_{0}=\varphi\left(x_{0}\right) \in \varphi(U)$ and sequence $\left\{y_{n}\right\} \subset Y \backslash \varphi(U)$ converging to $y_{0}$. By using (ii) it is easy to obtain a contradiction.

The authors are grateful to the referees for useful remarks and recommendations providing improvement of this paper.

The research of the first author is supported by the grants RFBR 08-01-00208, MD-2195.2008.1, and NSh-3233.2008.1, the second author is supported by the grants RFBR 07-01-00156 and 09-01-00424a and by the analytical departmental target program "Development of scientific potential of the higher school 2009-2010", grant 2.1.1/500.

\section{Bibliography}

[1] R.Phelps Lectures on Choquet's theorem, Springer, 2001.

[2] E.Alfsen, Compact convex sets and boundary integrals, Springer Verlag, 1971. 
[3] A.D.Joffe, W. M.Tikhomirov, Theory of extremum problems, AP, NY, 1979.

[4] G.A.Edgar, "Extremal integral representations", J. Functional Analysis, 23:2 (1976), $145-161$.

[5] G.A.Edgar, "On the Radon-Nikodim property and martingale convergence", Lecture Notes in Mathematics, 645 (1978), 62-76.

[6] R.D.Bourgin, Geometric aspects of convex sets with the Radon-Nikodiym property, Lecture Notes in Mathematics, 993, Springer-Verlag, Berlin, 1983.

[7] R.D.Bourgin, G.A.Edgar, "Noncompact simplexes in Banach spaces with the RadonNikodiym property", J. Functional Analysis, 23:2, (1976), 162-176.

[8] P.A.Meyer, Probability and potentials, Ginn (Blaisdell), Boston, 1966.

[9] M.E.Shirokov "On the strong CE-property of convex sets", Mathematical Notes, 82:3, (2007) 395-409;

[10] J.Vesterstrom, "On open maps, compact convex sets, and operator algebras", J. London Math. Soc. 6:2 (1973), 289-297.

[11] A.Lima, "On continuous convex functions and split faces", Proc. London Math. Soc., 25:3 (1972), 27-40.

[12] R.O'Brien, "On the openness of the barycentre map" Math. Ann., 223:3 (1976), 207212.

[13] S.Papadopoulou, "On the geometry of stable compact convex sets" Math.Ann. 229 (1977), 193-200.

[14] A.Clausing, S.Papadopoulou, "Stable convex sets and extremal operators", Math.Ann., 231 (1978), 193-203.

[15] R.Grzaslewicz, "Extreme continuous function property", Acta.Math.Hungar. 74 (1997), 93-99.

[16] E.S.Polovinkin, M.V.Balashov, Elements of convex and strongly convex analysis, Fizmatlit, ., 2004 (in Russian).

[17] V.I.Bogachev, Foundations of measure theory, RKhD, Moscow-Izhevsk 2003 (in Russian).

[18] K.Parthasarathy, Probability measures on metric spaces, Academic Press, New York and London, 1967.

[19] N.N.Vahania, V.I.Tarieladze "Covariant operators of probability measures in locally convex spaces", Theory of Probability and its Applications, V.23, N.1, P.1-23, 1978. 
[20] M.E.Shirokov, "The characterization of convex $\mu$-compact sets", Russian Mathematical Surveys, 63:5 (2008), 981-982.

[21] M.E.Shirokov, A.S.Holevo "On approximation of infinite-dimensional quantum channel", Probl. Inf. Transm., 44:2 (2008), 73-90.

[22] A.S.Holevo, M.E.Shirokov "Continuous ensembles and the $\chi$-capacity of infinite dimensional channels", Theory of Probability and its Applications, 50:1 (2005), 86-98.

[23] I.C.Gohberg, M.G.Krein, Theory of linear non-Hermitian operators in Hilbert space, M.Nauka, 1965.

[24] L.Q.Eifler, "Open mapping theorems for probability measures on metric spaces", Pacific Journal of Mathematics, 66 (1976), 89-97.

[25] M.E.Shirokov, "The Holevo capacity of infinite dimensional channels and the additivity problem", Commun. Math. Phys., 262:1 (2006), 137-159.

[26] A.S.Holevo, M.E.Shirokov, R.F.Werner "On the notion of entanglement in Hilbert space", Russian Math. Surveys, 60:2 (2005), 359-360.

[27] A.S.Holevo Introduction to quantum information theory, MCCME, 2002 (in Russian).

[28] G.Vidal, "Entanglement monotones", J.Mod.Opt. 47 (2000) 355-376.

[29] M.B.Plenio, S.Virmani, "An introduction to entanglement measures", arXiv: quant-ph/0504163 (2005).

[30] T.J. Osborne "Convex Hulls of Varieties and Entanglement Measures Based on the Roof Construction", Quantum Information and Computation, 7:3 (2007), 209-227.

[31] P.Rungta, C.M.Caves, "Concurrence-based entanglement measures for isotropic states", Phys. Rev. A, 67:1 (2003), 012307/1-9.

[32] S.Boyd, L.Vandenberghe, Convex optimization, Cambridge Univ. Press. 2006. 